\title{
Diversity structure of the microbial communities in the guts of four neotropical termite species
}

Surendra Vikram ${ }^{1}$, Joel D. Arneodo ${ }^{2}$, Javier Calcagno ${ }^{3}$, Maximiliano Ortiz $^{1}$, Maria Laura Mon ${ }^{2}$, Clara Etcheverry $^{4}$, Don A. Cowan ${ }^{1}$, Paola Talia ${ }^{\text {Corresp. } 2}$

${ }^{1}$ Centre for Microbial Ecology and Genomics, Department of Biochemistry, Genetics and Microbiology, University of Pretoria, Pretoria, Gauteng, South Africa

2 Instituto de Agrobiotecnología y Biología Molecular (IABIMO), Instituto Nacional de Tecnología Agropecuaria (INTA), Consejo Nacional de Investigaciones Científicas y Técnicas (CONICET), Hurlingham, Buenos Aires, Argentina

3 Centro de Ciencias Naturales, Ambientales y Antropológicas, Universidad Maimonides. (CCNAA), CABA, Argentina

4 Biología de los Invertebrados, Facultad de Ciencias Exactas y Naturales y Agrimensura. Universidad Nacional del Nordeste, Corrientes, Argentina

Corresponding Author: Paola Talia

Email address: talia.paola@inta.gob.ar

The termite gut microbiome is dominated by lignocellulose degrading microorganisms. This study describes the intestinal microbiota of four Argentinian higher termite species with different feeding habits: Microcerotermes strunckii (hardwood), Nasutitermes corniger (softwood), Termes riograndensis (soil organic matter/grass) and Cornitermes cumulans (grass) by deep sequencing of amplified 16S rRNA and ITS genes. In addition, we have performed a taxonomic and gut community structure comparison incorporating into the analysis the previously reported microbiomes of additional termite species with varied diets. The bacterial phylum Spirochaetes was dominant in the guts of $M$. strunckii, $N$. corniger and $C$. cumulans, whereas Firmicutes predominated in the $T$. riograndensis gut microbiome. A single bacterial genus, Treponema (Spirochaetes), was dominant in all termite species, except for $T$. riograndensis. Both in our own sequenced samples and in the broader comparison, prokaryotic $\alpha$-diversity was higher in the soil/grass feeders than in the wood feeders. Meanwhile, the $\beta$-diversity of prokaryotes and fungi was highly dissimilar among strict wood-feeders, whereas that of soil- and grass- feeders grouped more closely. Ascomycota and Basidiomycota were the only fungal phyla that could be identified in all gut samples, because of the lack of reference sequences in public databases. In summary, higher microbial diversity was recorded in termites with more versatile feeding sources, providing further evidence that diet, along with other factors (e. g. host taxonomy), influences the microbial community assembly in the termite gut. 


\section{Diversity structure of the microbial communities in the guts of four}

\section{Neotropical Termite Species}

3

4 Surendra Vikram ${ }^{1}$, Joel D. Arneodo ${ }^{2}$, Javier Calcagno $^{3}$, Maximiliano Ortiz $^{1}$, Maria Laura Mon²,

5 Clara Etcheverry ${ }^{4}$, Don A. Cowan ${ }^{1}$ and Paola Talia ${ }^{2 *}$

6

$7 \quad{ }^{1}$ Centre for Microbial Ecology and Genomics, Department of Biochemistry, Genetics and

8 Microbiology, University of Pretoria, Pretoria, South Africa.

$9{ }^{2}$ Instituto de Agrobiotecnología y Biología Molecular (IABIMO), Instituto Nacional de

10 Tecnología Agropecuaria (INTA), Consejo Nacional de Investigaciones Científicas y Técnicas

11 (CONICET), Hurlingham, Buenos Aires, Argentina.

$12{ }^{3}$ Centro de Ciencias Naturales, Ambientales y Antropológicas, Universidad Maimonides.

13 (CCNAA), CABA, Argentina.

$14{ }^{4}$ Biología de los Invertebrados. Facultad de Ciencias Exactas y Naturales y Agrimensura.

15 Universidad Nacional del Nordeste, Corrientes, Argentina.

$16 *$ Corresponding author. Tel: +54 1146211447 Int 3627

17 E-mail address: talia.paola@inta.gob.ar/taliapaolam@gmail.com 
24 Abstract

25 The termite gut microbiome is dominated by lignocellulose degrading microorganisms. This study describes the intestinal microbiota of four Argentinian higher termite species with different feeding habits: Microcerotermes strunckii (hardwood), Nasutitermes corniger (softwood), Termes riograndensis (soil organic matter/grass) and Cornitermes cumulans (grass) by deep sequencing of amplified 16S rRNA and ITS genes. In addition, we have performed a taxonomic and gut community structure comparison incorporating into the analysis the previously reported microbiomes of additional termite species with varied diets. The bacterial phylum Spirochaetes was dominant in the guts of M. strunckii, N. corniger and C. cumulans, whereas Firmicutes predominated in the T. riograndensis gut microbiome. A single bacterial genus, Treponema (Spirochaetes), was dominant in all termite species, except for $T$. riograndensis. Both in our own sequenced samples and in the broader comparison, prokaryotic $\alpha$-diversity was higher in the soil/grass feeders than in the wood feeders. Meanwhile, the $\beta$-diversity of prokaryotes and fungi was highly dissimilar among strict woodfeeders, whereas that of soil- and grass- feeders grouped more closely. Ascomycota and Basidiomycota were the only fungal phyla that could be identified in all gut samples, because of the lack of reference sequences in public databases. In summary, higher microbial diversity was recorded in termites with more versatile feeding sources, providing further evidence that diet, along with other factors (e.g. host taxonomy), influences the microbial community assembly in the termite gut. 
54

55

56

57

58

59

60

61

62

63

64

65

66

67

68

69

70

71

74 72 Syntermitinae and Termitinae. To date, 80 genera and 458 species, distributed in four 73 subfamilies, Apicotermitinae, Nasutitermitinae, Syntermitinae and Termitinae, have been

\section{Introduction}

Termites are extremely efficient in degrading lignocellulose, and may be useful as "bioreactor models" for the conversion of lignocellulosic biomass into biofuels and other biomaterials (Brune, 2014).

Termites are broadly separated into 'lower' and 'higher' groups. The gut microbiota of lower termites consists of Bacteria, Archaea and Eucarya (such as flagellates and yeasts), whereas higher termites lack flagellated protozoans (Ni \& Tokuda, 2013). These microbial symbionts have various roles in digestive processes, by participating in multiple functions including carbohydrate and nitrogen metabolism, oxygen and hydrogen consumption, $\mathrm{N}_{2}$ fixation, modifications of aromatic polymers and humification (Brune, 2014; Santana et al., 2015).

All termites feed on lignocellulose, the main component of plant cell walls. Lower termites (families Mastotermitidae, Kalotermitidae, Termopsidae, Hodotermitidae, Rhinotermitidae and Serritermitidae) have specific diets restricted to woody tissue, whereas higher termites (family Termitidae) have diverse feeding habits, which include wood, grass, fungi, lichen, litter, dung, humus and soil. Termitidae is the most diverse family of termites (around $75 \%$ of all species). This family comprises eight subfamilies: Apicotermitinae,

71 Cubitermitinae, Foraminitermitinae, Macrotermitinae, Nasutitermitinae, Sphaerotermitinae, 74 identified in the Neotropical Region (Krishna et al., 2013). 

phylogeny, with influence from the diet (Hongoh, 2010; Rahman et al., 2015; Tai et al., 2015).

More recently, Mikaelyan et al. (2015) suggested that the diet was the principal determinant of the higher termite gut microbiome composition showing that in all analysis of bacterial community structure, wood-feeding species were clearly separated from humus and soil feeders. Nevertheless, for each feeding source, a grouping of bacterial phylotypes by termite subfamily related to the taxonomy of the host was evidenced (Mikaelyan et al. 2015). Furthermore, Calusinska et al. (2020) investigated the adaptation of two higher termite colonies

(Cortaritermes sp.) to Miscanthus (a perennial grass) consumption on laboratory conditions and constated the development of a diet-driven, adapted microbial consortium. Most authors agree that insect gut bacterial diversity is determined by environmental behavior, diet, developmental stage and host phylogeny (Hoback \& Stanley, 2001; Yun et al. 2014; Rahman et al. 2015; Bourguignon et al., 2018; Dietrich et al., 2014). However, the relative influence of each of these factors is still not fully elucidated. In line with the above-mentioned studies, Rahman et al. (2015) concluded that even though the termite gut microbiome is mainly modulated by vertical inheritance, there may be adaptative changes in the microbial populations due to diet. Also, Dietrich et al. (2014) showed that phylogeny is not the unique factor influencing the termite microbiota composition, as they observed that changes in the diet or new niches can modify the bacterial community structure. Bourguignon et al. (2018) stated that termite gut microbiota is a result of a combination of vertical inheritance showing strong host specificity and horizontal transmission, where the latter can occur indirectly through the feeding substrates or via aggressive encounters. In addition, other authors conclude that there is a functional correlation between gut microbiomes from different termite hosts (Marinowska et al., 2020). They affirm 
98 that each termite species is a unique organism with its own gut microbiome and that there are

99 functional similarities between microbial populations across different termite hosts.

In this study, we used prokaryotic 16S rRNA gene and fungal internal transcribed spacer

101 (ITS) sequences to compare the gut microbiota of four higher termite species with different

102 feeding habits and from three different subfamilies Cornitermes cumulans (Syntermitinae),

103 Microcerotermes strunckii (Termitinae, Amitermes group), Nasutitermes corniger

104 (Nasutermitinae) and Termes riograndensis (Termitinae, Termes group). Cornitermes cumulans

105 builds mounds and feeds mainly on herbaceous material, predominantly grasses, but its diet may

106 include cow dung and degraded wood (Souza et al., 2017). The soil-mound building termite $T$.

107 riograndensis feeds mainly on soil and plant material. On the other hand, $M$. strunckii and $N$.

108 corniger are strict wood -feeders, and therefore consume dry, wet or partially decayed

109 hardwoods and softwoods (Scheffrahn et al., 2005). Here, we determined the structure of the

110 bacterial and fungal communities in their guts and we performed a taxonomic and gut

111 community structure comparison among these and other termite species previously reported.

112 This is the first characterization of the gut microbiota of T. riograndensis and M. strunckii. Thus, 113 this research provides novel information on the gut microbial communities of some unexplored

114 termite species and contributes to shed light on the ecology and evolution of termites and their 115 gut symbionts.

117 Materials \& Methods

118 Insect collection

119 The termite species Cornitermes cumulans, Microcerotermes strunckii, Nasutitermes corniger 120 and Termes riograndensis are widely distributed in Northeastern Argentina. 

notatum Flüggé. M. strunckii (S 2742'43.9": W 59¹3'35.2") and N. corniger (S 27²7'38.3":

Santin (hardwood) and Peltophorum dubium (Spreng.) Taub (softwood), respectively.

endangered or protected species were used in this study. The taxonomic identification of the

Worker specimens were stored at $-20^{\circ} \mathrm{C}$ until further processing.

\section{DNA extraction}

134 Worker caste specimens were surface sterilized with $70 \%$ ethanol and their whole guts were dissected under a binocular microscope using sterile forceps. Ten dissected whole guts were pooled in a microtube containing RNA-later (Ambion, Grand Island, USA); three independent extractions were performed per termite species. Microbial genomic DNA was extracted from the triplicate gut samples using the DNeasy Blood and Tissue kit (Qiagen, USA) according to the manufacturer's instructions. In order to maximize the disruption of the gut tissues and their content, a thoroughly grinding with plastic pestles was performed prior to the chemical lysis. 
144 region of the ITS rDNA was amplified using the specific barcoded primers Forward (5'-

145 GCATCGATGAAGAACGCAGC-3') and Reverse (5'-

146 ATATGTAGGATGAAGAACGYAGYRAA-3') to assess fungal diversity. The samples were

147 sequenced on an Illumina MiSeq instrument at the Molecular Research DNA (MR DNA)

148 sequencing facility (Shallowater, Texas, USA).

\section{Bioinformatic and statistical analysis of $16 \mathrm{~S}$ rRNA and ITS sequences}

151 The paired end short reads were merged into single end reads and demultiplexed using the barcode sequences for each sample. The analyses of the 16S rRNA and ITS sequences were performed in the Qiime2 v2018.6 (https://qiime2.org) (Bolyen et al., 2019). Chimera identification and Amplicons Sequence Variants (ASVs) clustering were performed using

DADA2 (Callahan et al., 2016) plugin in QIIME 2. The sequences were trimmed from the left at 35 base pairs (to remove leftover adapter and primer sequences) and truncated at 232 base pairs during the ASV clustering in DADA2. A broader community structure comparison was performed, including our data and those obtained previously from seven additional termite species by downloading the published database sequences (Bourguignon et al., 2018; Mykaelyan et al., 2017) (Table 1). During the ASV clustering step sequences were trimmed 40 bases pairs from left $\left(5^{\prime}\right)$ (to remove any leftover adapter/primer sequence) and truncated at 200 base pairs during the DADA2 step. The sequences were assigned to ASVs using SILVA 128 16S rRNA (Quast et al., 2013) and UNITE (Abarenkov et al., 2010) database for Bacteria/Archaea and fungi, respectively. 
168 diversity indices for the 16S rRNA were analyzed in the QIIME 2 pipeline. In addition, $\alpha$ -

169 diversity between the termite gut microbiomes was compared using the Kruskal-Wallis test,

170 followed by false discovery rate (FDR) correction in Qiime2 pipeline. Weighted and unweighted

171 UniFrac dissimilarities matrices were also obtained from the QIIME 2 pipeline (Lozupone et al.,

172 2011). The Unifrac distances were plotted in phyloseq package (McMurdie \& Holmes, 2013)

173 using the Principal coordinate analysis (PCoA) (for four termite species from this work) and

174 Non-metric multidimensional scaling (NMDS) (for 11 termite species comparison). The

175 comparative analysis of $16 \mathrm{~S}$ rRNA ASV table rarefied for 14,042 sequences in 10,736 ASVs

176 (total number of sequences 393,176 for 27 samples). One sample corresponding to

177 Neocapritermes taracua was removed from the further downstream analyses due to low number

$178(8,210)$ of sequences obtained.

179 Alpha- and $\beta$ - diversity indexes for the ITS sequences (fungal communities) were

180 calculated and applied in the phyloseq (McMurdie \& Holmes, 2013) and Vegan (Dixon, 2003)

181 package in R. The test of significance for the $\beta$ - diversity of ITS ASVs was performed after

182 Hellinger transformation and Bray-Curtis distance matrix applied for the adonis function in

183 Vegan package. The test of homogeneity of dispersion was performed using the betadisper

184 function and Bray-Curtis dissimilarity in the Vegan package. The analysis of significant

185 differences was performed based on the groups (termite diet (soil/grass, humus, litter and wood))

186 and host phylogeny (different subfamilies within Termitidae). A $p$ value $<0.05$ was set as the

187 cutoff for significance of the statistical tests (The paired end short reads were merged into single

188 end reads and demultiplexed using the barcode sequences for each sample. The analyses of the

189 16S rRNA were performed in the Qiime2 v2018.6 (https://qiime2.org) (Bolyen et al., 2019). 
191

192

194

195

196

197

198

199

200

201

202

203

204

205

206

207

208

209

210

211

212

213

\section{Accession Numbers}

The sequences obtained in this study are available in NCBI Sequence Read Archive (Bioproject PRJNA480379). The accession numbers are SRR7503210 (16S rRNA gene) and SRR7503211 (ITS).

\section{Results}

Illumina MiSeq sequencing of 16S rRNA gene and ITS amplicons, derived from gut samples from four termite species generated 1,761,565 high quality sequences. The 16S rRNA ASV table was rarefied to 79,256 sequences resulting in 4,882 ASVs (total number of sequences 951,072 for 12 samples). The ITS ASV table was rarefied to 7,418 sequences belonging to 149 ASVs (from a total of 66,762 sequences obtained from nine samples) (Table S1A). In addition, in the comparative analysis of the results from this study with previous works, a total number of sequences 393,176 for 27 samples were included; the 16S rRNA ASV table was rarefied for 14,042 sequences in 10,736 ASVs (Table S1B).

\section{Bacterial, archaeal and fungal taxonomy}

We identified 23 bacterial phyla in the termite guts of the four species tested. The dominant communities in the guts of wood-feeding termites (M. strunckii and $N$. corniger) were Spirochaetes (51\% to $61 \%)$, followed by Fibrobacteres ( 13\%). Both species also showed similar relative abundances of Bacteroidetes $(\sim 8 \%)$ and Firmicutes $(\sim 8 \%$ in the case of $N$. corniger and slightly less, $\sim 6 \%$, for $M$. strunckii). Only a few reads $(\sim 2 \%)$ remained as unclassified bacteria (Fig. 1A). Thus, at the phylum level, gut communities of both wood-feeding species shared highly similar profiles regarding dominant taxa. 
For the grass-feeding termite C. cumulans, Spirochaetes was again the dominant phylum

215 ( $\sim 4 \%$ of the total bacterial community), followed by Firmicutes ( $23 \%)$ and Bacteroidetes

$216(\sim 13 \%)$. By contrast, the dominant phylum in the soil/grass-feeding species $T$. riograndensis was

217 Firmicutes $(\sim 32 \%)$, followed by Spirochaetes $(\sim 20 \%)$ and Bacteroidetes $(\sim 13 \%)$. Only $2 \%$ of the

218 sequences remained unclassified (Fig. 1A). Overall, Treponema was the most abundant bacterial

219 genus in M. strunckii (59.1\%), N. corniger (58.3\%) and C. cumulans (42.8\%). This genus was

220 also found in T. riograndensis, though at a lower relative abundance (17.5\%), where the genus

221 Lactococcus predominated.

222 Of the Archaea, the only two phyla that could be identified, Euryarchaeota and

223 Bathyarchaeota accounted for less than $2 \%$ of the reads, except for $T$. riograndensis, in which

224 they represented almost $6 \%$ on average.

225 We also compared the gut bacterial and archaeal taxonomy and community structures

226 found in this study with those of seven additional higher termites available in public databases. A

227 total of 35 bacterial/archeal phyla were identified in the termite guts of the 11 species analyzed.

228 Gut communities were dominated by Spirochaetes in the range of $10 \%$ to $74 \%$, Firmicutes $(4 \%$

229 to $63 \%)$, Bacteroidetes $(0.2 \%$ to $70 \%)$ and Fibrobacteres $(0.9 \%$ to $32 \%)$. Less abundant, though

230 still well represented were Proteobacteria $(<21 \%)$ and Actinobacteria $(<19 \%)$, among others

231 (Fig. S1). A little fraction of the reads could not be assigned to any phylum. As for the other

232 phyla, the relative abundance of the Spirochaetes was variable within and between the diet

233 groups. However, the highest abundance was observed in the wood-feeding termite group (up to

$23474 \%$ ) followed by the soil- and/or grass-feeding group (up to 49\%) and the litter-feeder termites

235 Cornitermes sp. (consistently $45 \%$ ). Termites that feed on soil and/or grass, and humus showed

236 high relative abundance of the phylum Firmicutes (up to $\sim 55 \%$ for both groups, compared to 
$237 \sim 15-25 \%$ for Cornitermes sp. and less than 14\% for wood-feeders). One of the triplicates of the

238 wood-feeder M. parvus exhibited an unexpectedly high proportion of Firmicutes, but this was

239 not corroborated in any of the other two replicates. At the genus level most dominant taxa were

240 Termite Treponema cluster followed by Treponema sp. regardless of the diet group (Fig. S2).

241 For the ITS analysis, C. cumulans was excluded because of the low number of reads obtained.

242 Regarding the other three termite species, the absence of matches with the available sequence

243 data in UNITE $(\sim 81 \%, \sim 93 \%$ and $\sim 99 \%$ of unclassified reads for $N$. corniger, $T$. riograndensis,

244 and M. strunckii, respectively) did not allow the taxonomic placement of most of the fungal

245 ASVs. The few taxa identified were assigned to the phyla Ascomycota and Basidiomycota, and

246 within the former, the class Eurotiomycetes was the most abundant (Fig. 1B).

247 A rarefaction analysis performed for each gut sequence dataset retrieved rarefaction

248 curves that reached a plateau for all samples, except for $C$. cumulans ITS sequences. This result

249 suggests that the sample size was large enough to represent the bacterial and fungal diversity

250 present in the communities (Fig. 2).

251

252 Diversity of prokaryotic and fungal taxa

253 The prokaryotic diversity of termite guts was analyzed using $\alpha$ - and $\beta$-diversity indices. The

254 indices, Shannon, Pielou's evenness and number of observed ASVs, showed no significant

255 differences in $\alpha$-diversity between the four newly reported termite microbiomes (Fig.

256 3).However, the $\alpha$-diversity between diet groups (strict wood -feeders and soil/grass -feeders)

257 significantly differed (Fig. S3). In addition, we evaluated the $\alpha$-diversity between diet groups

258 (soil/grass or strictly grass-feeders, humus -feeders, litter -feeders, and wood -feeders)

259 incorporating sequence data from public databases (Fig. 4). Soil/Grass or strictly grass diet group

260 showed highest number of observed ASVs, however, the comparison was only significantly

Peer] reviewing PDF | (2020:08:52210:2:0:NEW 21 Jan 2021) 
261 different for the diet groups soil/grass-humus (Krukal-Wallis; $\mathrm{H}=6.3, p=0.011, q=0.035$ ) and

262 soil/grass-wood (Kruskal-Wallis; $\mathrm{H}=7.7, p=0.005, q=0.031$ ) (Fig. 4C). The Pielou's-evenness

263 indices showed significant differences between the soil/grass-humus $(\mathrm{H}=8.0, p=0.004, q=0.008)$

264 and soil/grass-wood $(\mathrm{H}=9.2, p=0.002, q=0.007)$ groups. Also, a significant difference was

265 observed between the humus-litter $(\mathrm{H}=5.0, p=0.025, q=0.038)$ and humus-wood $(\mathrm{H}=10.0$, $266 p=0.001, q=0.007)$ groups (Fig. 4B).

267 The prokaryotic $\beta$-diversity of the termite gut microbiome was compared using the 268 unweighted UniFrac distances. The gut microbiome composition of the polyphagous soil/grass 269 feeders was found to be similar and grouped distantly from that of the wood feeders, which were, 270 in turn, separated from each other (Fig. 5A). The PERMANOVA test of Unifrac distances

271 revealed that the $\beta$-diversity was significantly different for the four termite species, with a

272 marked variation according to the diet group of termites (strict wood-feeders and soil/grass -

273 feeders) $(p<0.05)$ (Table 2$)$. Furthermore, the prokaryotic $\beta$-diversity of the new and database-

274 retrieved termite gut microbiomes were compared using the weighted and unweighted UniFrac

275 distances. Significant differences were observed on the unweighted UniFrac distances $(p<0.05)$,

276 although no differences were found with weighted UniFrac distances (Table 2). Though, when

277 considering diet groups, significant differences were found for both weighted $(p=0.005)$ and

278 unweighted $(p=0.001)$ Unifrac distances. The NMDS of unweighted Unifrac distances revealed

279 two separated clusters along the NMDS1 axis, the four gut microbiome studied in this work

280 grouped distantly from those reported by Mykaelyan et al. (2017) who in turn were separated

281 from those reported by Bourguignon et al. (2018) on the second axis (Fig 5B). The NMDS of

282 weighted Unifrac distances did not show grouping of the samples in the ordination plot based on

283 the diet groups (Fig 5C). The dissimilarity between the termite gut prokaryotic compositions for 
284 the diet group was compared using the betadisper (to test the homogeneity dispersion) and adonis

285 (to test the similarity between the prokaryotic communities). The distance to group centroids

286 based on Bray-Curtis distance were significantly different for diet groups (Betadisper, Permutest

$287 \mathrm{~F}=19.607, p=0.001)$. Adonis also showed significant differences $\left(\mathrm{F}_{(2.646)}=0.25, p=0.001\right)$ in the

288 community composition between the diet group of termites. However, when the termites were

289 grouped according to the subfamilies they belong to, no significant differences were observed

290 irrespective of the distance metric used (weighted or unweighted Unifrac) (Table 2).

291 The analysis of the fungal community structure was restricted to our novel sequence data,

292 since such information is still lacking in public databases. The Shannon and Pielou's evenness $\alpha-$

293 diversity indices of fungal communities from M. strunckii, N. corniger and T. riograndensis gut

294 samples differed significantly (Fig. S4). However, the number of observed ASVs showed no

295 significant differences in $\alpha$-diversity. Fungal $\alpha$-diversity according to the diet group was also

296 significantly different for the Shannon and Pielou's evenness indices but not for the number of

297 observed ASVs (Fig. S5).

298 To visualize overall similarities and differences in fungal community structure, we

299 calculated Bray-Curtis distances between M. strunckii, N. corniger, and T. riograndensis, and

300 displayed these analyses in the form of two-dimensional NMDS plots (Fig. S6). These analyses

301 revealed that the fungal community composition in the gut samples of the three termite species

302 was significantly different, whereas replicates of the same species were almost identical (Fig.

303 S6). PERMANOVA analysis confirmed significant differences in the fungal communities of the 304 different termite species and diet groups (Table 2).

305

306 Core microbiome 
307 In total, 38 bacterial ASVs were shared across the four higher termite species reported here;

308 which represent $28.5 \%$ of all the obtained sequences (Fig. 6A). The termite gut samples grouped

309 according to their feeding habits shared 23 (wood feeders) and 38 (soil/grass feeders) additional

310 ASVs. However, it has to be noted that $N$. corniger and T. riograndensis, which differ strongly

311 in their feeding source and belong to distinct subfamilies, shared 31 additional ASVs (Fig. 6A).

312 Of the 38 ASVs, 18 were assigned to Treponema sp., which represented 19\% of the core

313 microbiome sequences obtained (Fig. S6). These core ASVs were present in higher relative

314 abundances in the guts of C. cumulans, M. strunckii and M. corniger than in the guts of $T$.

315 riograndensis (Table S2; Fig S7).

316 The analysis of core prokaryotic communities incorporating a larger number of host

317 species (i.e. A. meridionalis, T. hospes, Cornitermes sp., Microcerotermes sp., M. parvus and

318 Nasutitermes sp.) revealed no shared ASVs between the four diet groups of termites (Fig 6B).

319 However, the soil/grass-wood groups shared 188 ASVs. A high number of ASVs were unique to

320 the termite species included in each diet group (soil/grass or grass: 3,729; humus: 2,046; liter:

321 1,285; wood: 3,379) (Fig 6B).

322 We identified 11 fungal core ASVs, which represented an average of $71.3 \%$ of all the

323 identified fungal sequences, shared in the gut samples of M. strunckii, N. coringer and T.

324 riograndensis (Fig. 6C). However, the most abundant shared ASVs could not be assigned to any 325 taxonomic level (Table S2).

327 Discussion

328 Numerous studies on the gut microbiota of higher termites have been published in the last two

329 decades (Warnecke et al., 2007; Otani et al., 2014; Mikaelyan et al., 2015; Rahman et al., 2015; 
330 Santana et al., 2015; Su et al., 2016, Bourguignon et al., 2018). Knowing the factors that shape

331 the structure of the microbial communities has become an increasing focus of interest. In this

332 sense, relative few studies have dealt with the gut community composition in relation to the

333 feeding habits of the host termite species.

334 This study provides a description of the intestinal microbiota associated with four

335 Argentinian higher termite species (C. cumulans, M. strunckii, N. corniger and T. riograndensis),

336 performed by high-throughput 16S rRNA gene and ITS amplicon sequencing analyses. Although

337 data exist on the bacterial microbiota of C. cumulans and N. corniger (Dietrich et al., 2014;

338 Köhler et al., 2012; Warnecke et al., 2007; Burnum et al., 2011; Mikaelyan et al., 2014; Costa et

339 al., 2013, Grieco et al., 2013; 2019); this is the first characterization of the gut microbiota of $T$.

340 riograndensis and $M$. strunckii. Furthermore, very little is known about the fungal community in

341 the microbiome of these and other termite species. These termites have different diet preferences

342 ranging from hard- and softwood, to herbaceous materials and, soil/grass. Compared to

343 softwood, hardwood harbors higher amounts of carbon content consisting on cellulose,

344 hemicellulose and low proportion of lignin (Demirbas, 2005). Herbaceous plant materials have

345 higher nutritious contents and lower lignin than wood, whereas soil contains diverse organic

346 matter that is selectively utilized by the termite species. We explored the gut microbiota

347 composition of the above-mentioned Argentinian higher termite species taking into consideration

348 their different diet preferences.

349 Overall, Spirochaetes, followed by Fibrobacteres, Bacteroidetes and Firmicutes were the

350 dominant gut phyla in termites feeding on wood or grass (M. strunckii, N. corniger and C.

351 cumulans). By contrast, the dominant phylum in the soil/grass feeder $T$. riograndensis was

352 Firmicutes, followed by Spirochaetes and Bacteroidetes. In accordance with our study, other 
353 researchers have reported Spirochaetes as one of the most abundant phyla in wood- and grass-

354 feeding higher termite guts (Hongoh et al., 2005; Warnecke et al., 2007; Köhler et al., 2012;

355 Brune, 2014; Dietrich et al., 2014; Mikaelyan et al., 2015; Rahman et al., 2015). In the last years

356 the majority of glycosyl hydrolase genes encoding putative cellulases and hemicellulases,

357 identified by metagenomic and metatranscriptomic studies, have been associated with

358 Spirochaetes, Fibrobacteres, Bacteroidetes and Firmicutes (Warnecke et al., 2007; He et al.,

359 2013; Ben Guerrero et al., 2015; Grieco et al., 2019; Calusinska et al., 2020; Marynowska et al.,

360 2020; Romero Victorica et al., 2020).

361 The high abundance of Spirochaetes (including the genus Treponema sp.), Fibrobacteres

362 and Bacteroidetes in wood-feeding termites may be related to the nitrogen fixation and

363 lignocellulosic processes (Lilburn et al., 2001; Breznak, 2002; Warnecke et al., 2007; Yamada et

364 al., 2007; Su et al., 2016). In addition, high abundance of Firmicutes (mostly Ruminococcacae)

365 in T. riograndensis was in accordance with previous reports of soil- and humus- feeding termites

366 (He et al., 2013; Dietrich et al., 2014; Mikaelyan et al., 2015; Santana et al., 2015).

367 In particular, several studies have reported a proportion of Spirochaetes of approximately

36850 to $60 \%$ of total prokaryotic population in the gut microbiome of $N$. corniger, similar to that

369 reported here (Warnecke et al., 2007; He et al., 2013; Dietrich et al., 2014; Santana et al., 2015;

370 Su et al., 2016). However, Köhler et al. (2012) observed a lower proportion of Spirochaetes in $N$.

371 corniger and $N$. takasagoensis. This discrepancy could be due to variations in DNA extraction

372 methods (Morgan et al., 2010) and/or the use of different PCR oligonucleotides (Engelbrektson

373 et al., 2010).

A low proportion of Archaea was present in the gut community profiles of the four higher

375 termites gut communities analyzed. The detected archaeal phyla were Euryarchaeota and 
376 Bathyarchaeota. Euryarchaeota includes closely related genera already known for their

377 methanogenic activity (Rahman et al., 2015). The methanogenic archaeon Methanimicrococcus

378 sp. was present in the core microbiome albeit in different proportions. This genus had already

379 been detected in the gut microbiomes of other higher termites and cockroaches (Paul et al., 380 2012).

381 The extremely high relative abundance of Treponema spp. (phylum Spirochaetes) in the 382 core of C. cumulans, M. strunckii and N. corniger, and at a lower extent in that of $T$.

383 riograndensis, suggests that Treponema genus has an important role on the overall physiology

384 and digestive processes of wood- and grass- feeding higher termites. The predominance of

385 Treponema in the termite gut microbiota has been pointed out by several authors (Warnecke et

386 al., 2007; Köhler et al., 2012; Shi et al., 2013; Benjamino \& Graf, 2016). Microbiome diversity

387 is thought to be mainly related to the phylogeny of the termite and also, to their diet habits. In

388 order to infer possible relationships between gut microbial community, phylogeny, and diet; the

389 gut communities of additional termite species reported elsewhere have been included for a

390 broader comparison. Again, Spirochaetes, Firmicutes, Bacteroidetes and Fibrobacteres were the

391 dominant phyla. Also the most dominant taxa were Termite Treponema cluster followed by

392 Treponema sp.

393 In the four species evaluated in this study, the diversity of the fungal community was

394 markedly lower than that of prokaryotes. The high proportion of taxonomically unclassified

395 fungal ASVs may result from the lack of representative sequences in the UNITE database

396 (Hongoh, 2010; Santana et al., 2015). Although fungi are not as prevalent as bacteria in higher

397 termite guts, an important unresolved issue is to determine the role of fungi in cellulolytic

398 processes development and fitness. Some of these functions could be to provide a nitrogen 
399

400

401

402

403

404

405

406

407

408

409

410

411

412

413

414

415

416

417

418

419

420

421

source, degrade high molecular weight molecules and produce pheromones for mating and communication (de León et al., 2016; Zhang et al., 2018). However, the role of fungal microbiota in these processes is not clear yet (Brune, 2014).

The fungi classes that could be identified in the core were Eurotiomycetes and Malasseziomycetes. Eurotiomycetidae are producers of secondary metabolites, fermentation agents and xerophile and psychrophile enzymes. They had been previously reported in the gut of the litter-feeding termite Synthermes wheeleri (Santana et al., 2015). Malasseziomycetes are ecologically diverse and wide spread yeasts. The genus Malassezia includes lipophilic yeasts and has been known as a common inhabitant of human skin (Paulino et al., 2008). A report by Zhang et al. (2003) also identified this yeast in the guts of beetles.

The presence of a common core microbiota suggests that these taxa are retained despite differences in habitat, geography and food source, and regardless of host phylogeny. This core composition may be important for the maintenance key functions and may serve as the basis for microbial community resistance and/or resilience (Huse et al., 2012; Shade \& Handelsman, 2012; Benjamino \& Graf, 2016). However, when sequences obtained from more termite species were added to the analysis, no shared ASVs between all four diet groups of termites was evident, suggesting that both host termite phylogeny and diet (and eventually other additional factors) can influence the community structures of gut microbiota.

The $\alpha$-diversity of gut bacterial communities in the soil/grass feeder group was significantly higher than that in wood feeders. Among the former group, the gut microbiota diversity in T. riograndensis (a soil- and grass- feeding termites) was higher than in species that fed on grass only (C. cummulans). The lower microbial diversity found in the wood- feeding termites may be related to the maintenance of a more specialized microbiota that is necessary for 
422 performing an efficient lignocellulose metabolism, and therefore for the host survival (Breznak

$423 \&$ Brune, 1994; Colman et al., 2012). The studied species of wood- feeding termites have a very

424 limited diet, which includes complex carbohydrates (cellulose, hemicellulose and lignin), and

425 this characteristic may explain the lower $\alpha$-diversity. Feeding on live trees may expose the host

426 and potentially its microbiota to tree physiological responses (Morewood et al., 2004), which

427 may further shape gut community dynamics. The higher $\alpha$-diversity in the soil/grass feeding

428 termites could be related to the diverse range of carbon and nitrogen sources available in their

429 diets; as more complex substrates require more complex degradative capacity and therefore more

430 complex communities. On the other hand, the host habitat also may influence the relative

431 bacterial abundances of the termite gut microbiota (Yun et al., 2014). When including in the

432 analysis previously reported sequences obtained from additional termite species, the bacterial $\alpha$ -

433 diversity indices (Shannon and Pielou's-evenness) and number of observed ASVs found in the

434 soil/grass or strictly grass diet group were, again, significantly higher than in wood-feeders.

435 Significant differences were also evidenced for some indices between the other feeding groups

436 suggesting that multiple factors, which may include diet and host taxonomy, contribute to shape

437 the gut microbiota.

438 The intercommunity analysis restricted to our four Argentinian termite species showed

439 that the gut microbiomes of soil/grass feeders were clearly separated from those of wood feeders.

440 Soil/grass-feeding termite species grouped closely, whereas the wood -feeders were spatially

441 separated from each other. The replicates of C. cumulans, T. riograndensis and M. strunckii

442 showed little variation, whereas those of the wood feeder $N$. corniger were more disperse. The

443 termites $M$. strunckii and N. corniger were sampled from alive trees of Myracrodruon balansae

444 (hardwood) and Peltophorum dubium (softwood). Even though the relative abundance at the 
445 phylum level was similar, microbial species composition was different between both termite 446 species.

The $\beta$-diversity analysis including previously reported gut microbiomes showed that the

448 differences between communities were due to the presence of distinct ASVs rather than to 449 changes in relative abundance. A permutation-based test on the unweighted Unifrac distances 450 showed significant differences between diets, although no differences were found with weighted 451 Unifrac distances. The same analysis applied to diet groups showed significant differences 452 among them. However, non-significant clustering of the termites regarding host taxonomy 453 (subfamilies) was observed. Although the unweighted Unifrac distances revealed two separated 454 clusters along the NMDS1 axis, these groupings are not related to diet or host phylogeny. 455 Test of homogeneity dispersion using betadisper suggested that the sample groups are 456 having significantly low variance in the community dispersal $(p<0.001)$. PERMANOVA test 457 using the adonis function also suggested the significant differences in the termite gut community 458 using the diet as a grouping factor. However, non-significant differences were observed grouping 459 by host phylogeny. The inter-host comparison among microbial communities based on 460 metagenomic data obtained by different research groups should be approached carefully. Besides methodological heterogeneity concerning microbial DNA extraction, amplification and analysis

462 (Morgan et al., 2010; Engelbrektson et al., 2010), several other issues, starting at insect 463 collection, are also to be considered. The specimen's provenance (e.g. substrate origin, single or 464 multiple colonies sampled, etc.), the number of pool replicates, to mention a few items, may 465 influence the results substantially. For instance, during a prospection of culturable Cohnellarelated bacteria in the guts of three Neotropical termites, the sequenced clones tended to group

467 according to their host species and to the different colonies from which insects were sampled, too 
468 (Arneodo et al., 2019). Thus, even though much data are available, a multiplicity of

469 environmental, methodological and technical variables makes a broad comparative analysis

470 difficult (Pollock et al., 2018). Some apparent inconsistencies between authors appear, and

471 analyses (especially those regarding diversity) may reflect not only the intrinsic characteristics of

472 the studied microbiotas but also the different experimental conditions.

473 The understanding of the termite-microbiome interaction requires the exploration of the

474 composition and structure of the microbiota, as well as the characterization of its main metabolic

475 activities in different taxonomic termite groups with different types of diets. Altogether, and

476 concerning the four Argentinian termite species, no obvious pattern was observed in the

477 microbial community structures, except for the similar relative abundance of bacterial phyla in

478 the case of strict wood- feeders. This provides further evidence that the gut microbiota

479 composition is the result of multiple factors, which may include (but not be limited to) diet and

480 host taxonomy.

481

\section{Conclusions}

483 We have explored prokaryotic and fungal community structures in the guts of four higher termite 484 species collected in NE Argentina: Nasutitermes corniger, Cornitermes cumulans, Termes

485 riograndensis and Microcerotermes strunkii. For the latter two species, this study constitutes the 486 first characterization of their associated microbiota. Also, we have performed a taxonomic and 487 gut community structure comparison incorporating into the analysis previously published 488 microbiome data sets of termites with different diet preferences. The bacterial phylum 489 Spirochaetes (in particular, the genus Treponema sp.), was dominant in the guts of M. strunckii, 490 N. cornigerand C. cumulans, whereas Firmicutes predominated in the T. riograndensis gut 
491 microbiome. In the broader analysis, also Spirochetes was the dominant phylum followed by

492 Firmicutes, Bacteroidetes and Fibrobacteres. Both in our own sequenced samples and in the

493

494

495

496

497

498

499

500

501

502

503

504

505

506

507

508

509

510

511

512

513

extensive comparison, prokaryotic $\alpha$-diversity was higher in the soil/grass feeders than in the

wood feeders. In addition, the $\beta$-diversity of prokaryotes and fungi was highly dissimilar

among strict wood-feeders, whereas that of soil- and grass- feeders grouped more closely.

However in the broad comparison the $\beta$-diversity analysis showed significant differences

regarding diets but non-significant clustering of the termites for the host phylogeny groups.

Concerning fungi, our work provides new insights on a poorly studied field. Ascomycota and

Basidiomycota were the only fungal phyla that could be identified in all gut samples, because of

the lack of reference sequences in public databases. This study provides evidence that

communities are shaped by multiple factors that may include, among others, diet and host taxonomy.

\section{Acknowledgements}

PT, JDA, JC and MLM are CONICET members. CE acknowledges the CONICET Fellowship. SV and MO acknowledge the postdoctoral research fellowships from the University of Pretoria, South Africa. The authors are grateful to Dr. Julia Sabio y García for linguistic improvement in the manuscript. This study was supported by grants from the Fondo Argentino de Cooperación Internacional -FOAR- (Ministerio de Relaciones Exteriores y Culto de Argentina) \#6530 and \#6745, the Instituto Nacional de Tecnología Agropecuaria (INTA) (PNAIyAV-1130034) and the Agencia Nacional de Promoción Científica y Tecnológica (ANPCyT) Proyectos de Investigación Científica y Tecnológica (PICT) 2018 No. 4149.

Peer) reviewing PDF | (2020:08:52210:2:0:NEW 21 Jan 2021) 


\section{Author Contributions}

515 Surendra Vikram performed the experiments, analyzed the data, prepared figures and tables,

516 authored or reviewed drafts of the paper, and approved the final draft.

517 Joel D. Arneodo conceived and designed the experiments, performed the experiments, analyzed

518 the data, authored or reviewed drafts of the paper, approved the final draft.

519 Javier Calcagno analyzed the data, authored and reviewed drafts of the paper, approved the final 520 draft.

521 Maximiliano Ortiz analyzed the data, reviewed drafts of the paper, and approved the final draft.

522 Maria Laura Mon prepared figures, analyzed the data, reviewed drafts of the paper, and approved

523 the final draft.

524 Clara Etcheverry performed the experiments, analyzed the data, reviewed drafts of the paper, and 525 approved the final draft.

526 Don A Cowan analyzed the data, authored and reviewed drafts of the paper, and approved the 527 final draft.

528 Paola Talia conceived and designed the experiments, performed the experiments, analyzed the 529 data, prepared figures and/or tables, authored and reviewed drafts of the paper, and approved the 530 final draft.

\section{Tables and Figures titles}

533 Table 1: Summary of the higher termites used in the current study, their taxonomy classification, 534 feeding groups and accession numbers of bacterial 16S rRNA amplicon libraries.

535 Table 2: PERMANOVA analysis of bacteria/archaea and fungi. 
536 Fig 1. Relative abundance of bacteria, archaea (phyla level) and fungi (class level) in the gut of

537 Neotropical termites.

538 Fig 2. Rarefaction curves for the prokaryotic and fungi observed ASVs at 97\% sequence identity.

539 Fig 3. Prokaryotic $\alpha$-diversity measures (A) Shannon, (B) Pielou's evenness and (C) number of 540 observed ASVs of the four species of termites. Comparisons were performed using the Kruskal-

541 Wallis followed by Benjamini \& Hochberg FDR correction.

542 Fig 4. Prokaryotic $\alpha$-diversity measures.

543 Fig. 5. Beta-diversity NMDS plot of Unifrac distances.

544 Fig 6. Venn diagram showing the distribution of shared ASVs across the termite gut.

546 Tables and Figures legends

547 Table 1:

$548 *$ Termites collected in this study.

549 aBased on the food types given for termite genera (Jones \& Eggleton, 2011).

550 based on the dietary information in Souza et al., (2017).

551 'Based on dietary information in French \& Ahmed (2011).

552 dBased on observations of Gontijo \& Domingos (1991).

553 Table 2: The statistical analysis of ITS sequence data were performed based on the Hellinger

554 transformation and Bray-Curtis distance-based dissimilarity matrix.

555 Fig 1. (A) 16S rRNA gene (B) ITS sequence based taxonomic distribution in triplicate gut 556 samples.

557 Fig 2. (A) Rarefaction curves of total 16S rRNA sequence counts of 11 termite species gut 558 microbiomes. (B) Rarefied sequence plot for the 16S rRNA of 11 termite species gut 559 microbiome showing sufficient coverage at 14,042 sequences per sample. (C) Rarefaction curves 560 of total ITS sequences for four termite species. (D) ITS sequences of the total ASVs and 
561 sequence counts showing the enough coverage at the 7,418 sequences per sample. Termite

562 species from this work are denoted by the red asterisk.

563 Fig. 4. (A) Shannon, (B) Pielou's evenness and (C) number of observed ASVs of the total

564 species of higher termites used to compare between diet groups. Comparisons were performed 565 using the Kruskal-Wallis followed by Benjamini \& Hochberg FDR correction. The H stats, $p$

566 value and corrected $p$ value $(q)$ is written on the top of the paired comparison between the diet 567 groups.

568 Fig 5. (A) PCoA plot of unweighted Unifrac distance. (B) NMDS plot of unweighted Unifrac 569 distance of 11 termite species gut microbiomes (C) NMDS plot of weighted Unifrac distance of 57011 termite species gut microbiomes. The green, red, yellow, brown colors are representing the 571 grass, humus, litter and wood diet groups respectively. The different shapes are representing the 572 different termite species. Termite species from this work are denoted by the red asterisk.

573 Fig 6. (A) Shared prokaryotic ASVs between four termite species (B) Shared prokaryotic ASVs 574 between diet groups of termites and (C) Shared fungal ASVs between the three termites.

576 Supporting information

577 Fig S1. Taxonomy plot for the termite samples grouped into diet groups. The plot is showing the 578 relative abundance of the prokaryotic phyla in the gut of termites.

579 Fig S2. Taxonomy stack bar-plot of the prokaryotic communities in the diet group. (A) Phylum 580 level taxonomic classification (B) Genus level taxonomic classification. Taxa less than $2 \%$ were 581 combined and named as a " $<2 \%$ abund.". 
582 Fig S3. Prokaryotic $\alpha$-diversity measures (Shannon, Pieolou's evenness and number of observed 583 ASVs) according to diet groups (strict wood-feeders versus soil/grass- feeders). Comparisons 584 were performed using the Kruskal-Wallis followed by FDR method in Qiime2.

585 Fig S4. Fungal $\alpha$-diversity measures (Shannon, number of observed ASVs and Pieolou's 586 evenness) in three species of termites. Comparisons were performed using Kruskal-Wallis 587 followed by FDR method in Qiime2.

588 Fig S5. Fungal $\alpha$-diversity measures (Shannon, number of observed ASVs and Pieolou's 589 evenness) according to diet groups (strict wood-feeders versus soil/grass- feeders). Comparisons 590 were performed using Kruskal-Wallis followed by FDR method in Qiime2.

591 Fig S6. NMDS plot for the three termite gut samples using Bray Curtis analysis for ITS 592 sequences.

593 Fig S7. Relative abundance of 38 core ASVs at classified taxa level in the gut microbiome of $C$. 594 cumulans, M. strunckii, N. corniger and T. riograndensis. Redder and lighter red indicate greater 595 and less abundance, respectively.

596 Table S1. Number of input and final number of reads for the 16S rRNA and ITS sequence data 597 (S1A). Number of input and output 16S rRNA sequences obtained in the comparative analysis 598 (S1B).

599 Table S2: Relative abundance (\%) of C. cumulans, $M$. strunckii, $N$ corniger and T. riograndensis 600 core microbiota.

601

602

References

603 Abarenkov K, Henrik Nilsson R, Larsson KH, Alexander IJ, Eberhardt U, Erland S, Høiland K, 604 Kjøller R, Larsson E, Pennanen T, Sen R, Taylor AFS, Tedersoo L, Ursing BM, Vrålstad T, 
605 Liimatainen K, Peintner U, Kõljalg U. 2010. The UNITE database for molecular identification of 606 fungi - recent updates and future perspectives. New Phytologist 186: 281-285. DOI:

607 10.1111/j.1469-8137.2009.03160.x.

608 Arneodo JD, Etcheverry C, Thebe T, Babalola OO, Godoy MC, Talia P. 2019. Molecular 609 evidence that cellulolytic bacterial genus Cohnella is widespread among Neotropical 610 Nasutitermitinae from NE Argentina. Revista Argentina de Microbiología 51:77-80. DOI: 611 10.1016/j.ram.2017.11.008.

612 Ben Guerrero E, Arneodo J, Bombarda Campanha R, Abrão de Oliveira P, Veneziano 613 LabateMT, Regiani Cataldi T, Cataldi A, Labate CA, Martins Rodrigues C, Talia P. 2015.

614 Prospection and evaluation of cellulolytic and hemicellulolytic enzymes using untreated and 615 pretreated biomass in two argentinean native termites. PLoS One 10:e0136573. DOI:

616 10.1371/journal.pone.0136573.

617 Benjamino J, Graf J. 2016. Characterization of the Core and Caste-Specific Microbiota in the 618 Termite, Reticulitermes flavipes. Frontiers in Microbiology 7: 171. DOI:

619 10.3389/fmicb.2016.00171.

620 Bolyen E, Rideout JR, Dillon MR, Bokulich NA, Abnet CC, Al-Ghalith GA, Harriet A, 621 Alm EJ, Arumugam M, Asnicar F, Bai Y, Bisanz JE, Bittinger K, Brejnrod A, Brislawn CJ, 622 Brown CT, Callahan BJ, Caraballo-Rodríguez AM, Chase J, Cope EK, Da Silva R, Diener C, 623 Dorrestein PC, Douglas GM, Durall DM, Duvallet C, Edwardson, CF, Ernst M, Estaki M, 624 Fouquier J, Gauglitz JM, Gibbons SM, Gibson DL, Gonzalez A, Gorlick K, Guo J, Hillmann B, 625 Holmes S, Holste H, Huttenhower C, Huttley GA, Janssen S, Jarmusch AK, Jiang L, Kaehler 626 BD, Bin Kang K, Keefe CR, Keim P, Kelley ST, Knights D, Koester I, Kosciolek T, Kreps J, 627 Langille MGI, Lee J, Ley R, Liu YX, Loftfield E, Lozupone C, Maher M, Marotz C, Martin BD, 
628 McDonald D, McIver LJ, Melnik AV, Metcalf JL, Morgan SC, Morton JT, Naimey AT, Navas629 Molina JA, Nothias LF, Orchanian SB, Pearson T, Peoples SL, Petras D, Preuss ML, Rasmussen 630 LB, Rivers A, Robeson II MS, Rosenthal P, Segata N, Shaffer M, Shiffer A, Sinha R, Song SJ, 631 JR, Swafford AD, Thompson LR, Torres PJ, Trinh P, Tripathi A, Turnbaugh PJ, Ul-Hasan S, van 632 der Hooft JJJ, Vargas F, Vázquez-Baeza Y, Vogtmann E, von Hippel M, Walters W, Wan Y, 633 Wang M, Warren J, Weber KC, Williamson CHD, Willis AD, Xu ZZ, Zaneveld JR, Zhang Y, 634 Zhu Q, Knight R, Caporaso JG

635 . 2019. Reproducible, interactive, scalable and extensible microbiome data science using QIIME 636 2. Nature Biotechnology. 37(8): 852-7. DOI: 10.1038/s41587-019-0209-9.

637 Bourguignon T, Lo N, Dietrich C, Šobotník J, Sidek S, Roisin Y, Brune A, Evans TA. 2018.

638 Rampant Host Switching Shaped the Termite Gut Microbiome. Current Biology 28(4): 649639 654.e2. DOI: 10.1016/j.cub.2018.01.035.

640 Breznak JA, Brune A. 1994. Role of microorganisms in the digestion of lignocellulose by 641 termites. Annual Review of Entomology 39: 453-487. DOI:

642 10.1146/annurev.en.39.010194.002321.

643 Breznak NA. 2002. Phylogenetic Diversity and Physiology of Termite Gut Spirochetes.

644 Integrative and Comparative Biology 42: 313-318. DOI: 10.1093/icb/42.2.313.

645 Brune A. 2014. Symbiotic digestion of lignocellulose in termite guts. Nature Reviews

646 Microbiology 12: 168-180. DOI: 10.1038/nrmicro3182.

647 Callahan BJ, McMurdie PJ, Rosen M, Han AW, Johnson AJ, Holmes SP. 2016. DADA2: High-

648 resolution sample inference from Illumina amplicon data. Nature Methods 13: 581-583. DOI:

649 10.1038/nmeth.3869.

Peer] reviewing PDF | (2020:08:52210:2:0:NEW 21 Jan 2021) 
650 Calusinska M, Marynowska M, Bertucci M, Untereiner B, Klimek D, Goux X, Sillam-Dussès D, 651 Gawron P, Halder R, Wilmes P, Ferrer P, Gerin P, Roisin Y, Delfosse P. 2020. Integrative omics 652 analysis of the termite gut system adaptation to Miscanthus diet identifies lignocellulose 653 degradation enzymes. Communications Biology 3(1): 275. DOI: 10.1038/s42003-020-1004-3. 654 Caporaso JG, Lauber CL, Walters WA, Berg-Lyons D, Huntley J, Fierer N, Owens SM, Betley J, 655 Fraser L, Bauer M, Gormley N, Gilbert JA, Smith G, Knight R. 2012.Ultra-high-throughput 656 microbial community analysis on the illumina HiSeq and MiSeq platforms. ISME Journal 6, 657 1621-1624. DOI: 10.1038/ismej. 2012.8

658 Colman DR, Toolson EC, Takacs-Vesbach CD. 2012. Do diet and taxonomy influence insect gut 659 bacterial communities?. Molecular Ecology 20: 5124-5137. DOI: 10.1111/j.1365660 294X.2012.05752.x.

661 Costa PS, Oliveira PL, Chartone-Souza E. Nascimento AMA. Phylogenetic diversity of 662 prokaryotes associated with the mandibulate nasute termite Cornitermes cumulans and its 663 mound. 2013. Biology and Fertility of Soils 49, 567-574 (2013). DOI: 10.1007/s00374-012664 0742-x.

665 de León AV-P, Sanchez-Flores A, Rosenblueth M, Martínez-Romero E. 2016. Fungal 666 community associated with Dactylopius (Hemiptera: Coccoidea: Dactylopiidae) and its role in 667 uric acid metabolism. Frontiers in Microbiology 7: 954. DOI: 10.3389/fmicb.2016.00954.

668 Demirbas A. 2005. Estimating of structural composition of wood and non-wood biomass 669 samples. Energy Sources 27: 761-767. DOI: 10.1080/00908310490450971.

670 Dietrich C, Köhler T, Brune A, Kohler T, Brune A. 2014. The cockroach origin of the termite 671 gut microbiota: patterns in bacterial community structure reflect major evolutionary events. 672 Applied and Environmental Microbiology 80(7): 2261-2269. DOI: 10.1128/AEM.04206-13. 
673 Dixon P. 2003. VEGAN, a package of R functions for community ecology. Journal of

674 Vegetarian Science 14: 927-930. DOI: 10.1111/j.1654-1103.2003.tb02228.x.

675 Engelbrektson A, Kunin V, Wrighton KC, Zvenigorodsky N, Chen F, Ochman H, Hugenholtz P.

676 2010. Experimental factors affecting PCR-based estimates of microbial species richness and

677 evenness. The ISME Journal 4: 642-647. DOI: 10.1038/ismej.2009.153.

678 French JRJ, Ahmed BM. 2011. Biomimicry of Termite Social Cohesion and Design to Inspire

679 and Create Sustainable System. www.Intechopen.com. DOI:10.5772/19350.

680 Gontijo TA, Domingos DJ. 1991. Guild distribution of some termites from cerrado vegetation in 681 south-east Brazil. J Trop Ecol 7:523-9.

682 Grieco MB; Lopes FAC, Oliveira LS, Tschoeke DA, Popov, CC, Thompson CC, Gonçalves LC, 683 Constantino R, Martins OB, Kruger RH, de Souza W,Thompson FL. 2019. Metagenomic 684 Analysis of the Whole Gut Microbiota in Brazilian Termitidae Termites Cornitermes cumulans, 685 Cyrilliotermes strictinasus, Syntermes dirus, Nasutitermes jaraguae, Nasutitermes aquilinus, 686 Grigiotermes bequaerti, and Orthognathotermes mirim. Current Microbiology 76(6):687-697.

687 DOI: 10.1007/s00284-019-01662-3.

688 Grieco MA, Cavalcante JJ, Cardoso AM, Vieira RP, Machado EA, Clementino MM, Medeiros

689 MN, Albano RM, Garcia ES, de Souza W, Constantino R, Martins OB. 2013. Microbial

690 community diversity in the gut of the South American termite Cornitermes cumulans (Isoptera:

691 Termitidae). Microbial Ecololgy. 65(1):197-204. DOI: 10.1007/s00248-012-0119-6.

692 He S, Ivanova N, Kirton E, Allgaier M, Bergin C, Scheffrahn RH, Kyrpides NC, Nikos C,

693 Warnecke F, Tringe SG, Hugenholtz F. 2013. Comparative Metagenomic and

694 Metatranscriptomic Analysis of Hindgut Paunch Microbiota in Wood- and Dung-Feeding Higher

695 Termites. PLoS ONE 8: e61126. DOI: 10.1371/journal.pone.0061126. 
696 Hoback WW, Stanley DW. 2001. Insects in hypoxia. Journal of Insect Physiology 47: 533-542.

697 DOI: 10.1016/s0022-1910(00)00153-0.

698 Hongoh Y. 2010. Diversity and Genomes of Uncultured Microbial Symbionts in the Termite 699 Gut. Bioscience, Biotechnology and Biochemistry 74: 1145-1151. DOI: 10.1271/bbb.100094.

700 Hongoh Y, Deevong P, Inoue T, Moriya S, Trakulnaleamsai S, Ohkuma M. 2005. Intra- and 701 interspecific comparisons of bacterial diversity and community structure support coevolution of 702 gut microbiota and termite host. Applied and Environmental Microbiology 71(11): 6590-6599. 703 DOI: 10.1128/AEM.71.11.6590-6599.2005.Huse SM, Ye Y, Zhou Y, Fodor AA. 2012. A core 704 human microbiome as viewed through 16S rRNA sequence clusters. PLoS ONE 7: e34242. DOI: 705 10.1371/journal.pone.0034242.

706 Jones DT, Eggleton P. Global biogeography of termites. In: Bignell DE, Roisin Y, Lo N. (eds). 707 2011. Biology of Termites: A Modern Synthesis. Dordrecht: Springer, 2011, 477-98.

708 Katoh K, Misawa K, Kuma K, Miyata T. 2002. MAFFT: a novel method for rapid multiple 709 sequence alignment based on fast Fourier transform. Nucleic Acids Research. 30: 3059-3066.

710 DOI: 10.1093/nar/gkf436.

711 Köhler T, Dietrich C, Scheffrahn RH, Brune A. 2012. High-Resolution Analysis of Gut

712 Environment and Bacterial Microbiota Reveals Functional Compartmentation of the Gut in 713 Wood-Feeding Higher Termites (Nasutitermes spp.). Applied and Environmental Microbiology 714 78: 4691-4701. DOI: 10.1128/AEM.00683-12.

715 Krishna K, Grimaldi DA, Krishna V, Engel MS. 2013. Treatise on the Isoptera of the world. 716 Bulletin of the American Museum of Natural History 377: 2704. 
717 Lilburn TG, Kim KS, Ostrom NE, Byzek KR, Leadbetter JR, Breznak JA. 2001. Nitrogen

718 fixation by symbiotic and free-living Spirochetes. Science 292: 2495-2498. DOI:

719 10.1126/science.1060281.

720 Lozupone C, Lladser ME, Knights D, Stombaugh J, Knight R. 2011. UniFrac: an effective

721 distance metric for microbial community comparison. The ISME Journal 5: 169-172. DOI:

722 10.1038/ismej.2010.133.

723 McMurdie PJ, Holmes S. 2013. Phyloseq: An R Package for Reproducible Interactive Analysis

724 and Graphics of Microbiome Census Data. PLOS ONE 8(4): e61217. DOI:

725 10.1371/journal.pone.0061217.

726 Marynowska M, Goux X, Sillam-Dussès D, Rouland-Lefèvre C, Halder R, Wilmes P, Gawron P,

727 Roisin Y, Delfosse P, Calusinska M. 2020. Compositional and functional characterisation of

728 biomass-degrading microbial communities in guts of plant fibre- and soil-feeding higher

729 termites. Microbiome 8(1): 96. DOI: 10.1186/s40168-020-00872-3.

730 Mikaelyan A, Dietrich C, Köhler T, Poulsen M, Sillam-Dussès D, Brune A. 2015. Diet is the

731 primary determinant of bacterial communitystructure in the guts of higher termites. Molecular

732 Ecology 24: 5284-5295. DOI: 10.1111/mec.13376.

733 Mikaelyan A, Meuser K, Brune A. 2017. Microenvironmental heterogeneity of gut

734 compartments drives bacterial community structure in wood- and humus-feeding higher termites.

735 FEMS Microbiology Ecology 93(1):fiw210. DOI: 10.1093/femsec/fiw210.

736 Morewood WD, Hoover K, Neiner PR, McNeil JR, Sellmer JC. 2004. Host tree resistance

737 against the polyphagouswood-boring beetle Anoplophora glabripenn. Entomologia

738 Experimentalis et Applicata 110(1): 79-86. DOI: 10.1111/j.0013-8703.2004.00120.x. 
739 Morgan JL, Darling AE, Eisen JA. 2010. Metagenomic sequencing of an in vitro-simulated

740 microbial community. PLoS ONE 5(4):e10209. DOI: 10.1371/journal.pone.0010209.

741 Ni J, Tokuda G. 2013. Lignocellulose-degrading enzymes from termites and their symbiotic

742 microbiota. Biotechnology Advances 31: 838-850. DOI: 10.1016/j.biotechadv.2013.04.005.

743 Otani S, Mikaelyan A, Nobre T, Hansen LH, Koné NA, Sørensen SJ, Aanen DK, Boomsma JJ,

744 Brune A, Poulsen M. 2014. Identifying the core microbial community in the gut of fungus-

745 growing termites. Molecular Ecology 23(18): 4631-4644. DOI: 10.1111/mec.12874.

746 Pollock J, Glendinning L, Wisedchanwet T, Watson M. 2018. The Madness of Microbiome:

747 Attempting To Find Consensus "Best Practice" for 16S Microbiome Studies

748 Applied and Environmental Microbiology 84 (7): e02627-17. DOI: 10.1128/AEM.02627-17.

749 Paul K, Nonoh JO, Mikulski L, Brune A. 2012. 'Methanoplasmatales': Thermoplasmatales-

750 related archaea in termite guts and other environments are the seventh order of methanogens.

751 Applied and Environmental Microbiology 78: 8245-8253. DOI: 10.1128/AEM.02193-12.

752 Paulino LC, Tseng CH, Blaser MJ. 2008. Analysis of Malassezia microbiota in healthy

753 superficial human skin and in psoriatic lesions by multiplex real-time PCR. FEMS Yeast

754 Research 8: 460-471. DOI: 10.1111/j.1567-1364.2008.00359.x.

755 Price MN, Dehal PS, Arkin AP. 2010. FastTree 2--approximately maximum-likelihood trees for

756 large alignments. PLoS ONE 5: e9490. DOI:10.1371/journal.pone.0009490.

757 Quast C, Pruesse E, Yilmaz P, Gerken J, Schweer T, Yarza P, Peplies J, Glöckner FO. 2013. The

758 SILVA ribosomal RNA gene database project: improved data processing and web-based tools.

759 Nucleic Acids Research 41: 590-596. DOI: 10.1093/nar/gks1219.

760 Rahman NA, Parks DH, Willner DL, Engelbrektson AL, Goffredi SK, Warnecke F, Scheffrahn

761 RH, Hugenholtz P. 2015. A molecular survey of Australian and North American termite genera 
762 indicates that vertical inheritance is the primary force shaping termite gut microbiomes.

763 Microbiome 3: 2-16. DOI: 10.1186/s40168-015-0067-8.

764 Romero Victorica M, Soria MA, Batista-García RA, Ceja-Navarro JA, Vikram S, OrtizM,

765 Ontañon O, Ghio S,Martínez-Ávila L, Quintero García OJ, Etcheverry C, Campos E, Cowan D,

766 Arneodo J, Talia PM. 2020. Neotropical termite microbiomes as sources of novel plant cell wall

767 degrading enzymes. Scientific Reports 10(3864). DOI: 10.1038/s41598-020-60850-5.

768 Santana RH, Catão EC, Lopes FA, Constantino R, Barreto CC, Krüger RH. 2015. The gut

769 microbiota of workers of the litter-feeding termite Syntermes wheeleri (Termitidae:

770 Syntermitinae): archaeal, bacterial, and fungal communities. Microbial Ecology 70: 545-556.

771 DOI: 10.1007/s00248-015-0581-z.

772 Scheffrahn RH, Krecek J, Szalanski AL, Austin JW. 2005. Synonymy of Neotropical Arboreasl

773 Termites Nasutitermes corniger and N. costalis (Isoptera: Termitidae: Nasutermitinae), with

774 Evidence from Morphology, Genetics, and Biogeography. Annals of the Entomological Society

775 of America 98: 273-281. DOI:10.1603/0013-8746(2005)098[0273:SONATN]2.0.CO;2.

776 Shade A, Handelsman J. 2012. Beyond the Venn diagram: the hunt for a core microbiome.

777 Environmental Microbiology 14: 4-12. DOI: 10.1111/j.1462-2920.2011.02585.x.

778 Shi W, Xie S, Chen X, Sun S, Zhou X, Liu L, Gao P, Kyrpides NC, No EG, Yuanet JS. 2013.

779 Comparative Genomic Analysis of the Microbiome of Herbivorous Insects Reveals Eco-

780 Environmental Adaptations: Biotechnology Applications. PLoS Genetics 9: 1-12. DOI:

781 10.1371/journal.pgen.100313.

782 Souza G, Dos Santos VC, de Figueirredo Gontijo N, Constantino R, de Oliveira Paiva E, Silva

783 G, Bahia AC, Mendonça Gomes F, de Alcantara Machado E. 2017. Morphophysiological study 
784 of digestive system litter-feeding termite Cornitermes cumulans (Kollar, 1832). Cell and Tissue 785 Research 368: 579-590. DOI: 10.1007/s00441-017-2584-1.

786 Su L, Yang L, Huang S, Su X, Li Y, Wang FQ, Wang E, Kang N, Xu J, Song A. 2016.

787 Comparative Gut Microbiomes of Four Species Representing the Higher and the Lower

788 Termites. Journal of Insect Science 16: 1-9. DOI: 10.1093/jisesa/iew081.

789 Tai V, James ER, Nalepa CA, Scheffrahn RH, Perlman SJ, Keeling PJ. 2015. The role of host 790 phylogeny varies in shaping microbial diversity in the hindguts of lower termites. Applied and 791 Environmental Microbiology 81: 1059-1070. DOI: 10.1128/AEM.02945-14.

792 Turner S, Pryer KM, Miao VP, Palmer JD.1999. Investigating deep phylogenetic relationships 793 among cyanobacteria and plastids by smalls ubunit rRNAsequence analysis. Journal of Eukaryot 794 Microbiology 46, 327-338. DOI: 10.1111/j. 1550-7408.1999.tb04612.x.

795 Warnecke F, Luginbühl P, Ivanova N, Ghassemian M, Richardson TH, Stege JT, Cayouette M, 796 Mchardy AC, Djordjevic G, Aboushadi N, Sorek R, Tringe SG, Podar M, Martin HG, Kunin V, 797 Dalevi D, Madejska J, Kirton E, Platt D, Szeto E, Salamov A, Barry K, Mikhailova N, Kyrpides 798 NC, Matson EG, Ottesen EA, Zhang X, Hernández M, Murillo C, Acosta LG, Rigoutsos I, 799 Tamayo G, Green BD, Chang C, Rubin EM, Mathur EJ, Robertson DE, Hugenholtz P, 800 Leadbetter JR. 2007. Metagenomic and functional analysis of hindgut microbiota of a wood801 feeding higher termite. Nature 450: 560-565. DOI: 10.1038/nature06269.

802 Yamada A, Inoue T, Noda Y, Hongoh H, Ohkuma M. 2007. Evolutionary trend of phylogenetic 803 diversity of nitrogen fixation genes in the gut community of wood-feeding termites. Molecular 804 Ecology 16: 3768-3777. DOI: 10.1111/j.1365-294X.2007.03326.x.

805 Yun JH, Roh SW, Whon TW, Jung MJ, Kim MS, Park DS, Yoon C, Nam YD, Kim YJ, Choi JH, 806 Kim JY, Shin NR, Kim SH, Lee WJ, Bae JW. 2014. Insect gut bacterial diversity determined by 
807 environmental habitat, diet, developmental stage, and phylogeny of host. Applied and

808 Environmental Microbiology 80: 5254-64. DOI: 10.1128/AEM.01226-14.

809 Zhang N, Su SO, Blackwell M. 2003. Microorganisms in the gut of beetles: evidence from

810 molecular cloning. Journal of Invertebrate Pathology 84: 226-233. DOI:

811 10.1016/j.jip.2003.10.002.

812 Zhang Z, Jiao S, Li X, and Li M. 2018. Bacterial and fungal gut communities of Agrilus mali at 813 different developmental stages and fed different diets. Scientific Reports 8: 15634. DOI:

814 10.1038/s41598-018-34127-x. 
Figure 1

Relative abundance of bacteria, archaea (phyla level) and fungi (class level) in the gut of Neotropical termites.

(A) 16S rRNA gene (B) ITS sequence based taxonomic distribution in triplicate gut samples.

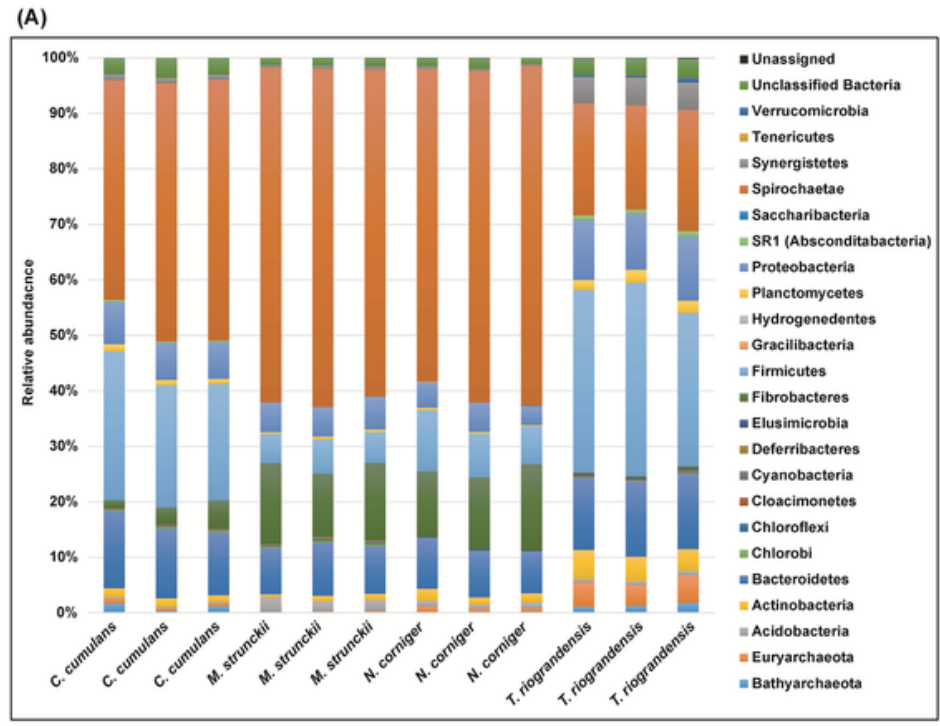

(B)

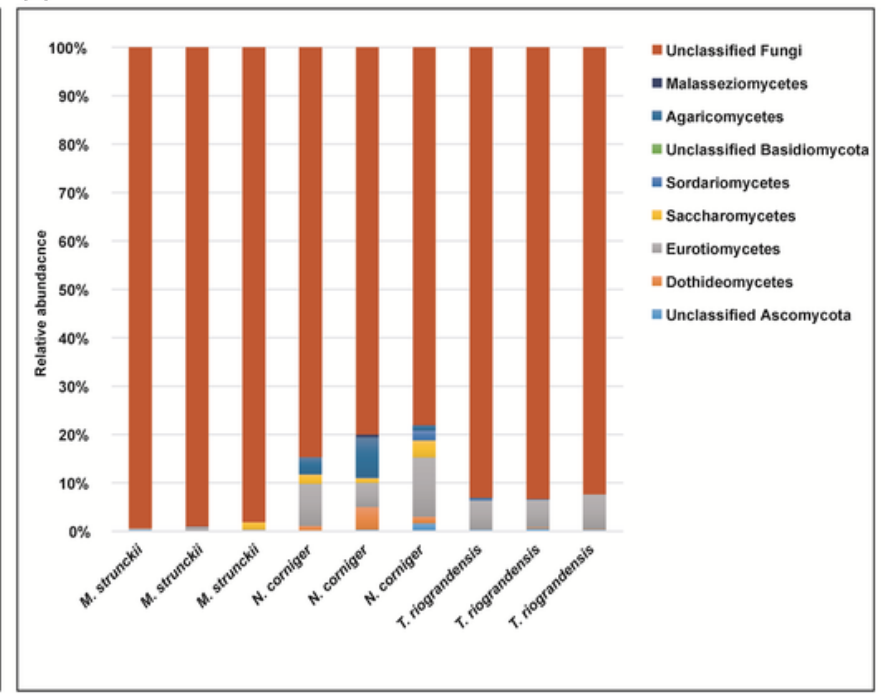




\section{Figure 2}

Rarefaction curves for the prokaryotic and fungi observed ASVs at $97 \%$ sequence identity.

(A) Rarefaction curves of total $16 \mathrm{~S}$ rRNA sequence counts of 11 termite species gut microbiomes. (B) Rarefied sequence plot for the 16S rRNA of 11 termite species gut microbiome showing sufficient coverage at 14,042 sequences per sample. (C) Rarefaction curves of total ITS sequences for four termite species. (D) ITS sequences of the total ASVS and sequence counts showing the enough coverage at the 7,418 sequences per sample. Termite species from this work are denoted by the red asterisk. 

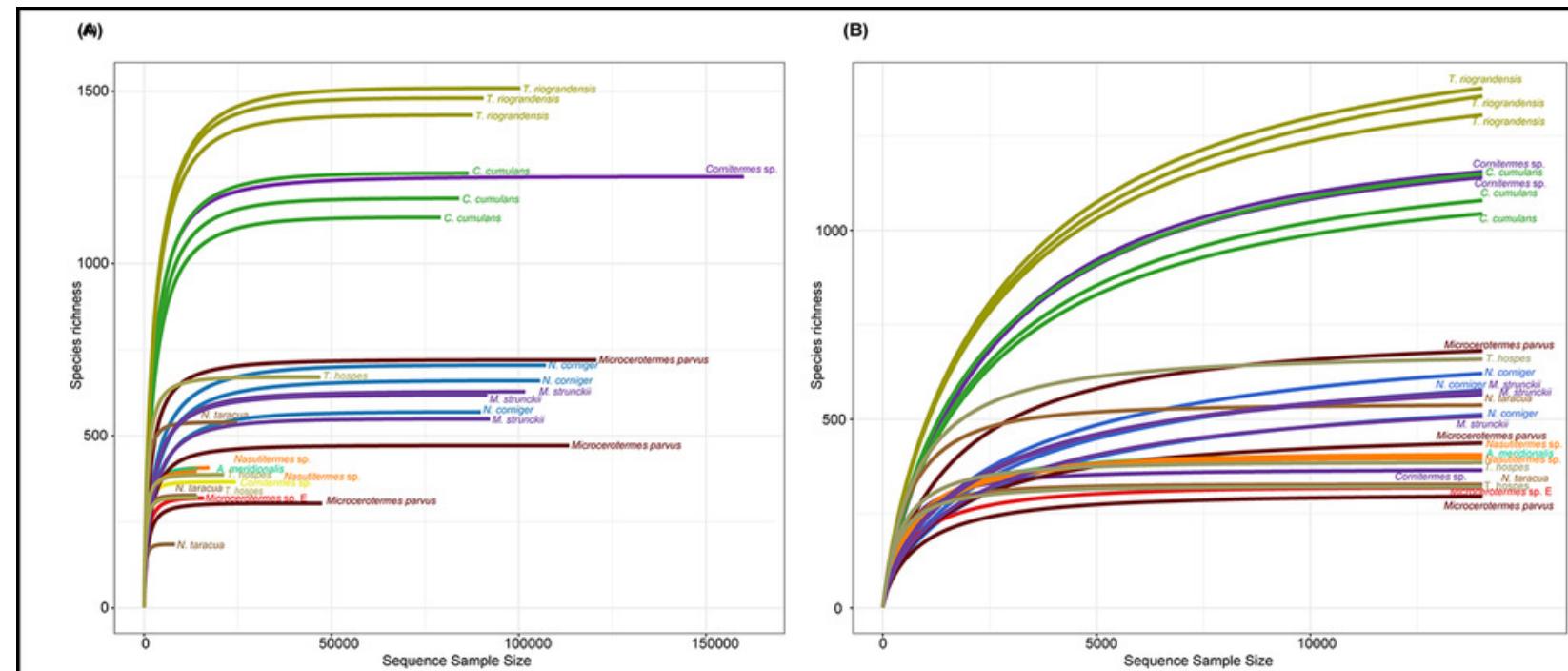

Termite species

- A mendionals

- Comitormos sp.

C

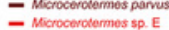

N. tarrous

- Nasudiomes sp.

$=$ M. strumckii*

(C)

(D)
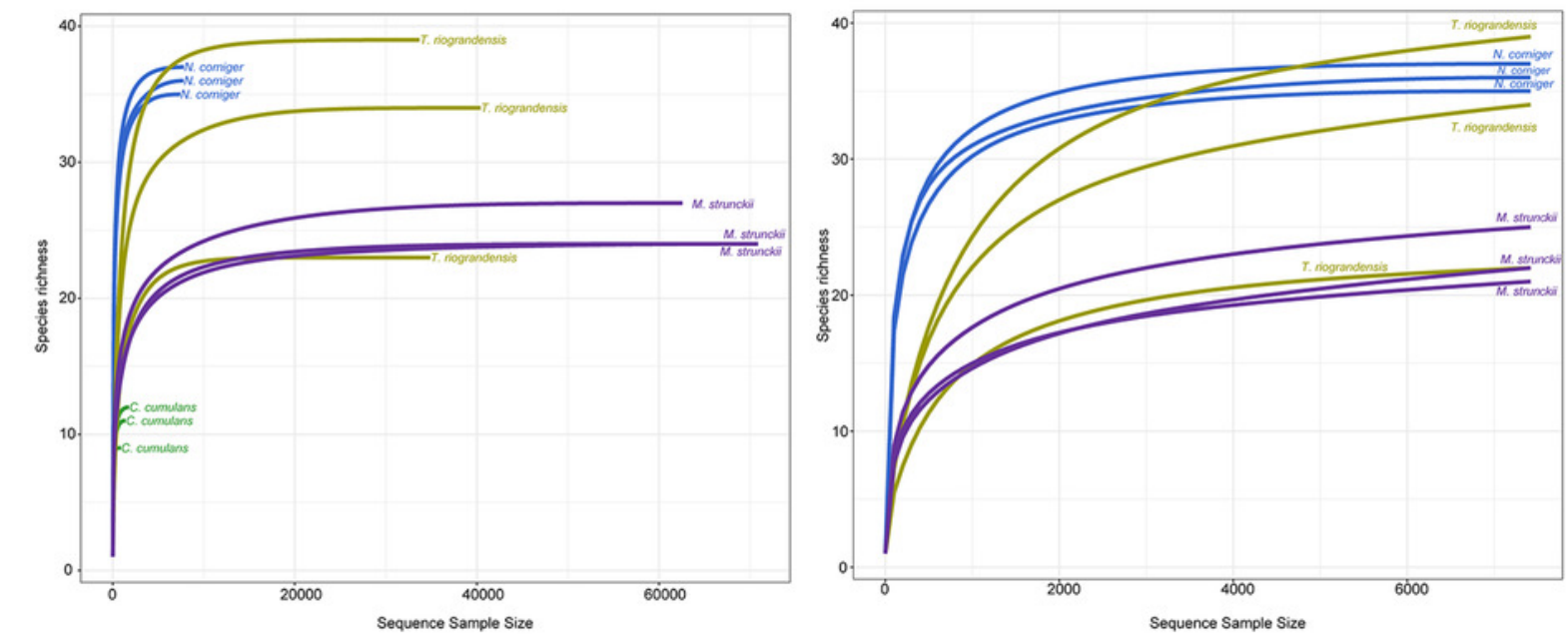

Termite species

$Z^{N}$ C. cominjort*

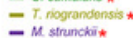


Figure 3

Prokaryotic $\alpha$-diversity measures.

(A) Shannon, (B) Pielou's evenness and (C) number of observed ASVs of the four species of termites. Comparisons were performed using the Kruskal-Wallis followed by Benjamini \& Hochberg FDR correction.
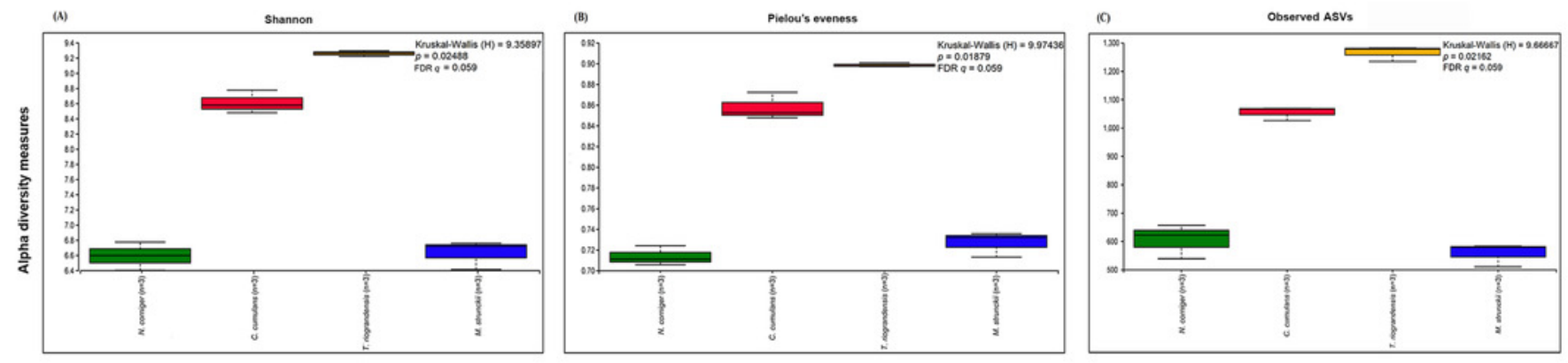
Figure 4

Prokaryotic $\alpha$-diversity measures.

(A) Shannon, (B) Pielou's e venness and (C) number of observed ASVs of the total species of higher termites used to compare between diet groups. Comparisons were performed using the Kruskal-Wallis followed by Benjamini \& Hochberg FDR correction. The $\mathrm{H}$ stats, $p$ value and corrected $p$ value $(q)$ is written on the top of the paired comparison between the diet groups.
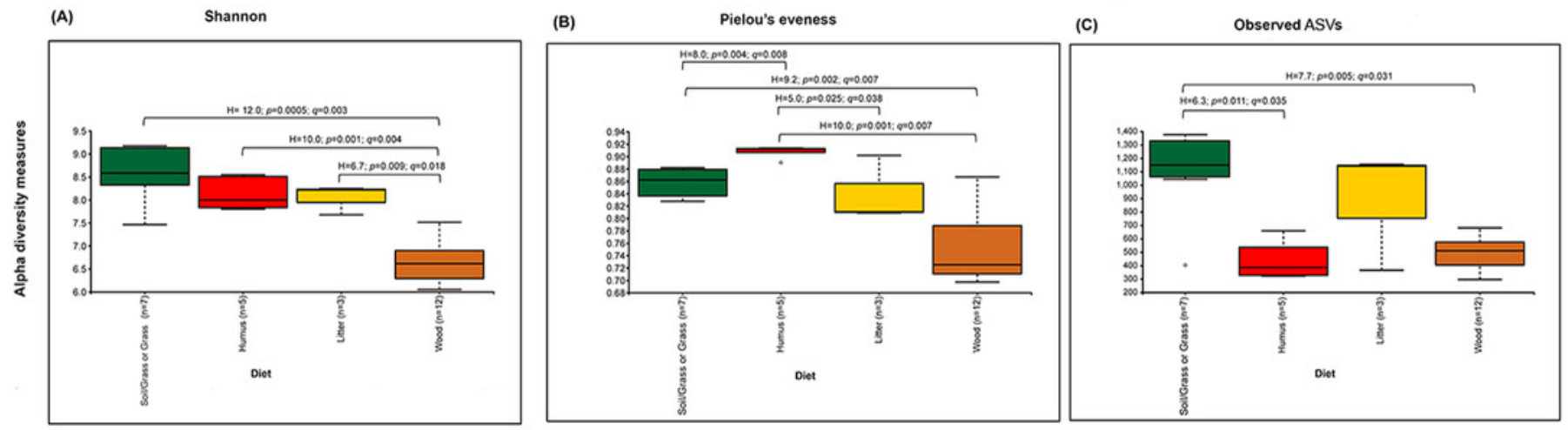
Figure 5

Beta-diversity NMDS plot of Unifrac distances.

(A) PCoA plot of unweighted Unifrac distance. (B) NMDS plot of unweighted Unifrac distance of 11 termite species gut microbiomes (C) NMDS plot of weighted Unifrac distance of 11 termite species gut microbiomes. The green, red, yellow, brown colors are representing the grass, humus, litter and wood diet groups respectively. The different shapes are representing the different termite species. Termite species from this work are denoted by the red asterisk

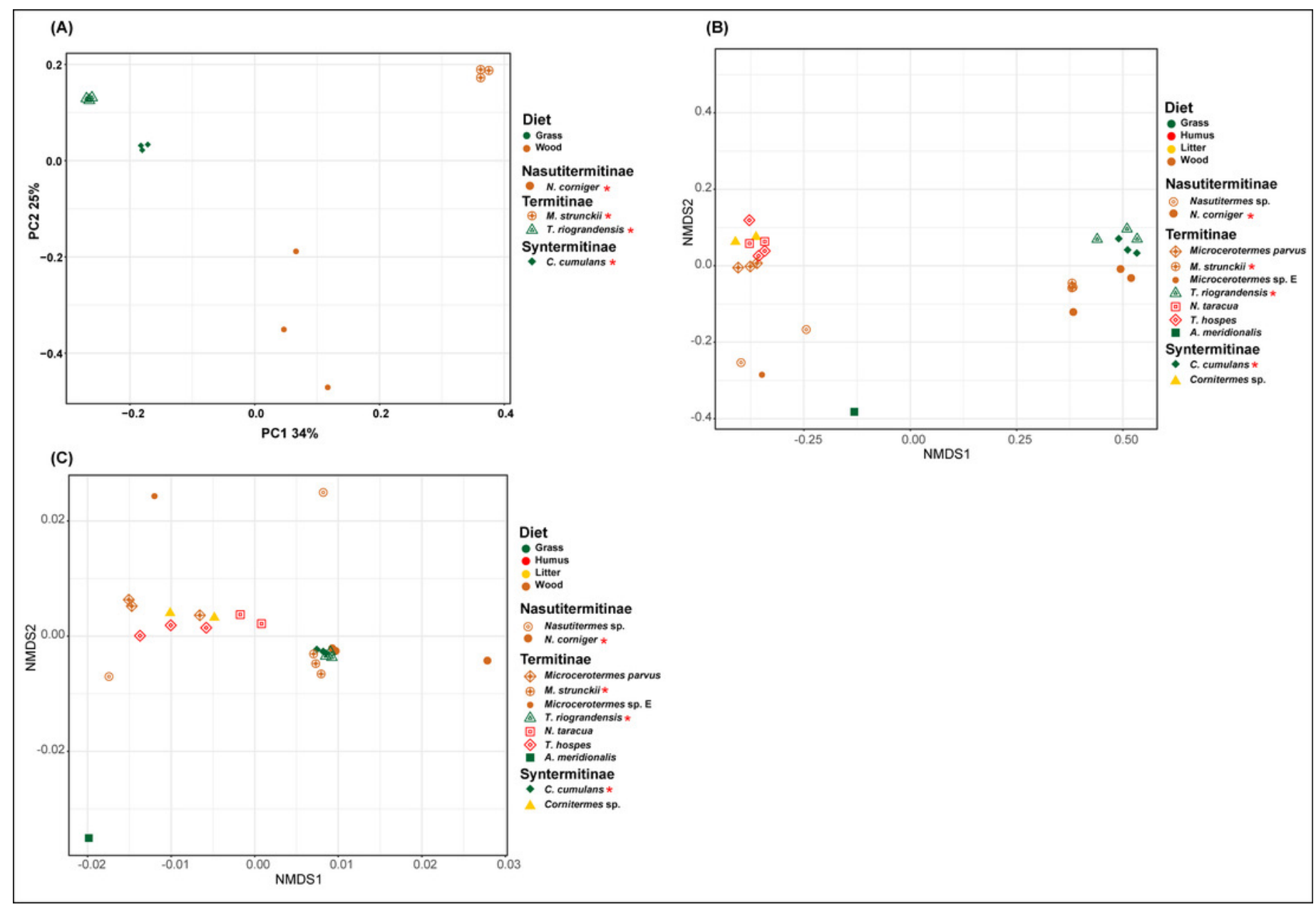


Figure 6

Venn diagram showing the distribution of shared ASVs across the termite gut.

(A) Shared prokaryotic ASVs between four gut microbiomes (B) Shared prokaryotic ASVs

between four gut microbiomes (B) Shared prokaryotic ASVs between diet groups of termites and (C) Shared fungal ASVs between the three termites. 
(A)

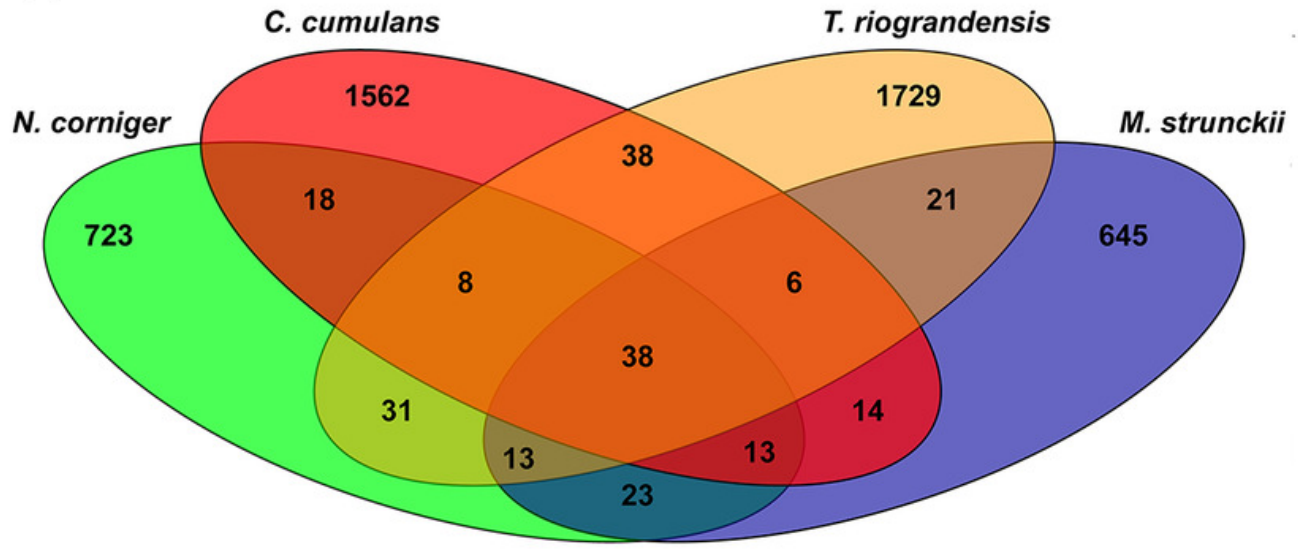

(B)

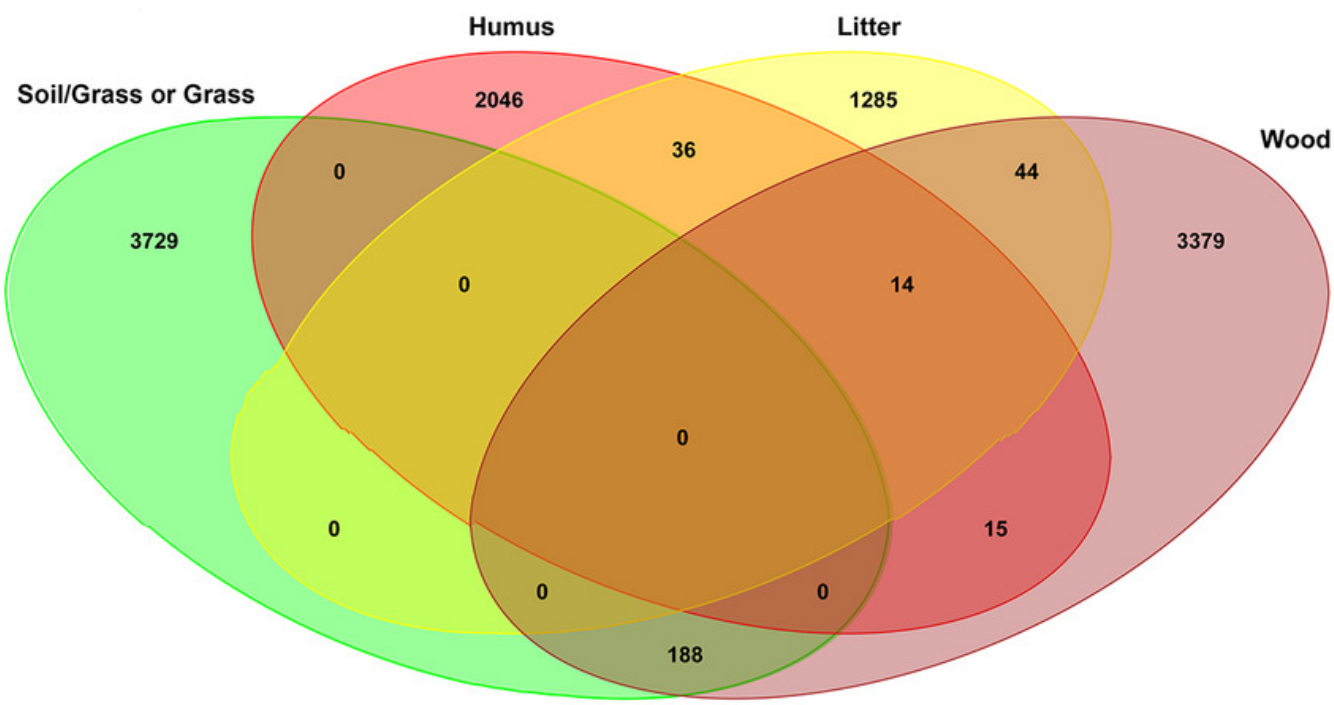

C. cumulans *

T. riograndensis

A. meridionalis

- T. hospes

Cornitermes sp.

- N. corniger *

- M. strunckii

Microcerotermes sp.

M. parvus

- Nasutitermes sp.

(C)

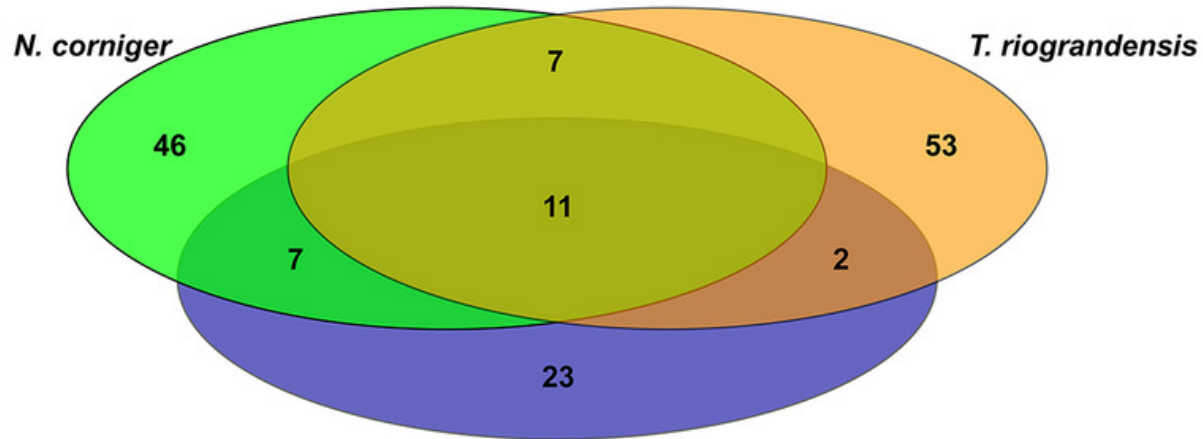

M. strunckii 


\section{Table $\mathbf{1}$ (on next page)}

Summary of the higher termites used in the current study, their taxonomy classification, feeding groups and accession numbers of bacterial 16S rRNA amplicon libraries.

*Termites collected in this study.

${ }^{\mathrm{a} B a s e d}$ on the food types given for termite genera (Jones \& Eggleton, 2011).

${ }^{\mathrm{b} B}$ Based on the dietary information in Souza et al., (2017).

'Based on dietary information in French \& Ahmed (2011).

${ }^{\mathrm{d} B a s e d}$ on observations of Gontijo \& Domingos (1991). 
1

2

\begin{tabular}{|c|c|c|c|c|c|}
\hline Host species & Subfamily & $\begin{array}{l}\text { Diet } \\
\text { preferences }\end{array}$ & Replicates & NCBI Biosample ID & References \\
\hline Nasutitermes corniger* & Nasutitermitinae & Wood $^{\mathrm{a}}$ & $(n=3)$ & SAMN09635494 & This work \\
\hline Nasutitermes sp. & Nasutitermitinae & Wood $^{\mathrm{a}}$ & $(n=2)$ & SAMN08180495 & Bourguignonet al., 2018 \\
\hline Microcerotermes sp. E & Termitinae & Wood $^{\mathrm{a}}$ & $(n=1)$ & SAMN08180514 & Bourguignon et al., 2018 \\
\hline $\begin{array}{l}\text { Microcerotermes } \\
\text { strunckii* }\end{array}$ & Termitinae & Wood $^{\mathrm{a}}$ & $(n=3)$ & SAMN09635494 & This work \\
\hline $\begin{array}{l}\text { Microcerotermes parvus } \\
\text { Mp193 }\end{array}$ & Termitinae & Wood $^{\mathrm{a}}$ & $(n=3)$ & SAMN04317068-70 & Mykaelyan et al., 2017 \\
\hline Cornitermes cumulans* & Syntermitinae & Grass $^{\mathrm{b}}$ & $(n=3)$ & SAMN09635494 & This work \\
\hline Amitermes meridionalis & Termitinae & Grass $^{\mathrm{c}}$ & $(\mathrm{n}=1)$ & SAMN08180513 & Bourguignon et al., 2018 \\
\hline Termes riograndensis* & Termitinae & Soil/Grass & $(n=3)$ & SAMN09635494 & This work \\
\hline $\begin{array}{l}\text { Neocapritermes taracua } \\
\text { Nt197 }\end{array}$ & Termitinae & Humus $^{\mathrm{a}}$ & $(n=2)$ & SAMN04317074-76 & Mykaelyan et al., 2017 \\
\hline Termes hospes Th196 & Termitinae & Humus $^{\mathrm{a}}$ & $(n=3)$ & SAMN04317083-85 & Mykaelyan et al., 2017 \\
\hline Cornitermes sp. Co191 & Syntermitinae & Litter $^{\mathrm{a}}$ & $(n=3)$ & SAMN04317065-67 & Mykaelyan et al., 2017 \\
\hline
\end{tabular}




\section{Table 2 (on next page)}

PERMANOVA analysis of bacteria/archaea and fungi.

The statistical analysis of ITS sequence data were performed based on the Hellinger transformation and Bray-Curtis distance-based dissimilarity matrix. 


\begin{tabular}{|c|c|c|c|c|}
\hline \multicolumn{3}{|c|}{ 16S rRNA } & & \\
\hline & \multicolumn{2}{|c|}{ Four termite species (This work) } & \multicolumn{2}{|c|}{11 termite species } \\
\hline & Unweighted Unifrac & $\begin{array}{l}\text { Weighted } \\
\text { Unifrac }\end{array}$ & $\begin{array}{l}\text { Unweighted } \\
\text { Unifrac }\end{array}$ & Weighted Unifrac \\
\hline Pseudo F & 8.5445 & 67.0377 & 1.5075 & 1.5407 \\
\hline \multirow[t]{2}{*}{$p$ value } & 0.001 & 0.001 & 0.031 & 0.199 \\
\hline & \multicolumn{2}{|c|}{ Diet groups (Grass and Wood) } & \multicolumn{2}{|c|}{$\begin{array}{l}\text { Based on diet groups (Grass, Humus, } \\
\text { Litter, and Wood) }\end{array}$} \\
\hline Pseudo F & 4.5241 & 12.4488 & 3.74797 & 5.37108 \\
\hline \multirow[t]{2}{*}{$p$ value } & 0.002 & 0.002 & 0.001 & 0.005 \\
\hline & & & \multicolumn{2}{|c|}{ Based on termite subfamily } \\
\hline Pseudo F & & & 1.61667 & 0.511453 \\
\hline$p$ value & & & 0.097 & 0.568 \\
\hline \multicolumn{5}{|c|}{ ITS (Bray-Curtis distance dissimilarity matrix) } \\
\hline & \multicolumn{2}{|c|}{ Four termite species (This work) } & & \\
\hline Pseudo F & 13.1240 & & & \\
\hline \multirow[t]{2}{*}{$p$ value } & \multicolumn{2}{|l|}{0.005} & & \\
\hline & \multicolumn{2}{|c|}{ Diet groups (Grass and Wood) } & & \\
\hline Pseudo F & \multirow{2}{*}{\multicolumn{2}{|c|}{3.8839}} & & \\
\hline$p$ value & & & & \\
\hline
\end{tabular}

